\title{
Large Magellanic Cloud stellar clusters
}

\section{21 HST colour magnitude diagrams ${ }^{\star \star \star \star}$}

\author{
E. Brocato ${ }^{1,2}$, E. Di Carlo ${ }^{1,3}$, and G. Menna ${ }^{1}$ \\ 1 Osservatorio Astronomico di Collurania, Via M. Maggini, 64100 Teramo, Italy \\ 2 Istituto Nazionale di Fisica Nucleare, LNGS, L'Aquila, Italy \\ 3 Area di ricerca in Astrogeofisica, L'Aquila, Italy \\ e-mail: brocato, dicarlo@astrte.te.astro.it
}

Received 22 June 2000 / Accepted 9 March 2001

\begin{abstract}
We present WFPC2 photometry of 21 stellar clusters of the Large Magellanic Cloud obtained on images retrieved from the Hubble Space Telescope archive. The derived colour magnitude diagrams (CMDs) are presented and discussed. This database provides a sample of CMDs representing, with reliable statistics, simple stellar populations with a large spread of age. The stars in the core of the clusters are all resolved and measured at least down to the completeness limit; the magnitudes of the main sequence terminations and of the red giant clump are also evaluated for each cluster, together with the radius at half maximum of the star density.
\end{abstract}

Key words. Galaxy: globular clusters: general - galaxies: evolution - galaxies: Magellanic Clouds

\section{Introduction}

The Large Magellanic Cloud (LMC) offers a unique opportunity to investigate bright populous stellar systems spanning a wide range of age and located at a distance short enough to gather photometric data of individual stars.

Pioneering studies by Hodge (1960a,b), Gascoigne (1966), Arp (1967), Robertson (1974) derived the colour magnitude (CM) diagrams of several LMC clusters providing valuable constraints on the stellar evolution theory of massive and intermediate mass stars (see for example Iben 1967; Becker \& Matthews 1983).

Up to now, a number of CMDs have been obtained for specific scientific purposes, in order, e.g., i) to investigate the efficiency of the overshooting mechanism (Da Costa et al. 1985; Chiosi et al. 1989; Brocato et al. 1989; Vallenari et al. 1994), ii) to study the red giant branch phase transition of LMC stellar clusters (Corsi et al. 1994) and the existence of a gap in their age distribution (Sarajedini

Send offprint requests to: E. Brocato,

e-mail: brocato@astrte.te.astro.it

* Based on observations made with the NASA/ESA Hubble Space Telescope, obtained from the data archive at the Space Telescope Institute. STScI is operated by the association of Universities for Research in Astronomy, Inc. under the NASA contract NAS 5-26555.

** Table 1 is only available in electronic form at http://www. edpsciences.org
1998), iii) to evaluate the age of the oldest clusters in this galaxy (Walker 1989, 1990, 1992, 1993a,b; Testa et al. 1995; Brocato et al. 1996; Olsen et al. 1998) and iv) to study the luminosity function of MC clusters (Mateo 1988).

Since these studies had different scientific targets, the $\mathrm{CM}$ diagrams available in the literature are derived from different telescopes and observing strategies, and do not share uniform data reduction techniques. As a result, we still lack homogeneous sample of CM diagrams of LMC clusters spanning a wide range of ages.

In this paper we present photometric data obtained from images retrieved from the Hubble Space Telescope (HST) archive providing CM diagrams for 21 LMC clusters, which improve the quality of the previous photometry, the number of stars and the limiting magnitude. Few clusters are also presented as a reference to recent ground measurements (NGC 2257; Walker 1992; Testa et al. 1995) and to compare independent data reduction procedures starting from the same observational data (NGC 2121, NGC 2155 and SL 663; Sarajedini 1998). As discussed in Sect. 3, one SMC cluster, Kron 3, is also included.

The main goal of this paper is to make available to the astronomical community homogeneous sample of CM diagrams of LMC clusters which can be used to investigate the properties (age, IMF etc.) of these objects in the general context of the history of the LMC and also to 

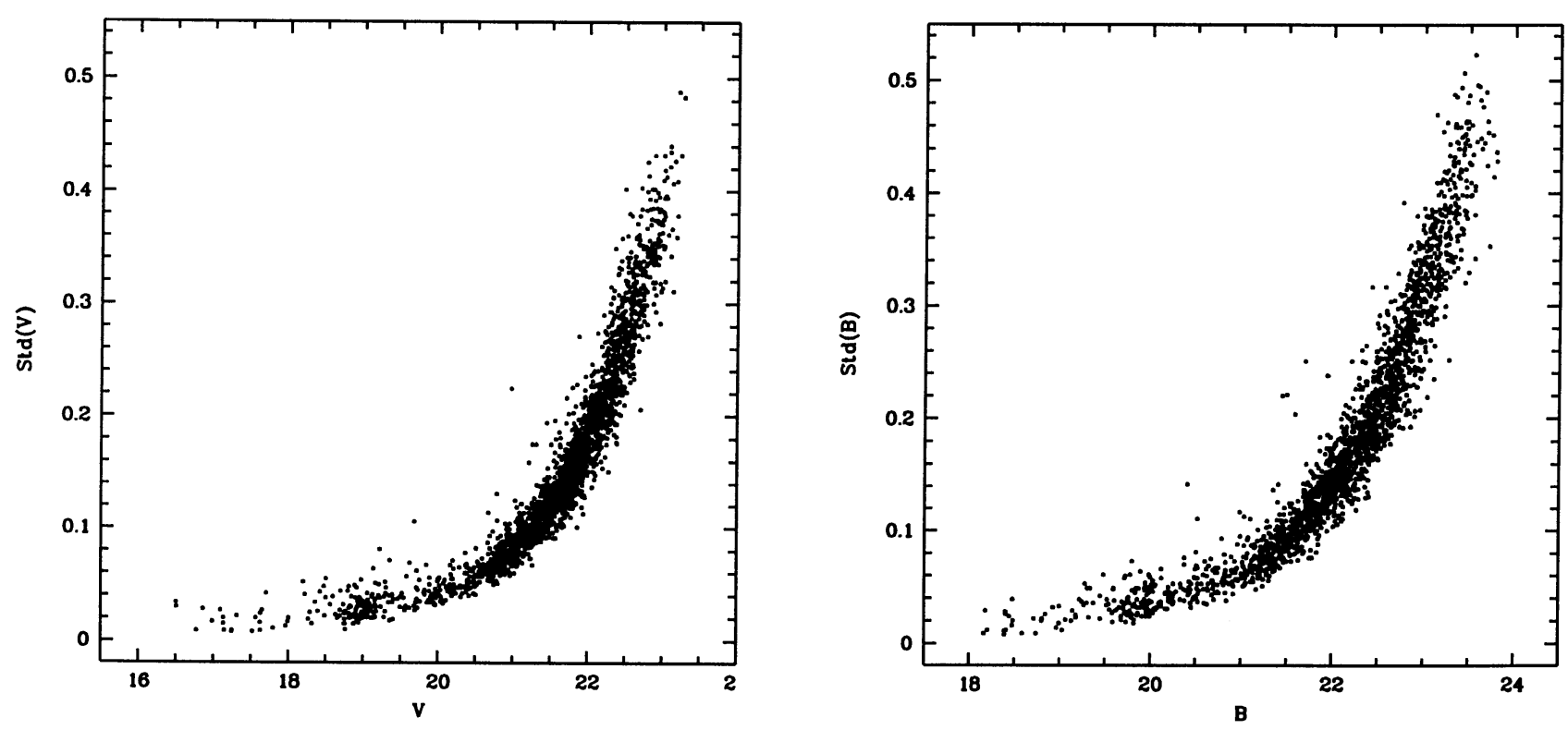

Fig. 1. The typical photometric uncertainties as obtained by DAOPHOT for the cluster NGC 2155.

probe the reliability and the accuracy of stellar evolution models.

In Sect. 2 we describe the photometric data, the $\log$ of the observations and the data reduction procedures adopted in this work. The CM diagrams are presented in Sect. 3, where their major features are also pointed out. Finally, in Sect. 4 some concluding remarks are briefly drawn.

\section{The HST archive images and the data reduction procedures}

The stellar clusters studied in this work were observed with the HST Wide Field and Planetary Camera 2 (WFPC2) in the F450W $(\approx B)$ and F555W $(\approx V)$ filters and with the gain value set at $14 e^{-} \mathrm{ADU}^{-1}$. The basic information on the images, as retrieved from the HST archive, are summarized in Table 1.

The observations are automatically processed through the standard STScI pipeline for bias removal and flat fielding.

To restore the true positions of the stars in the chip, the procedure by Holtzman et al. (1995a) is applied. The resulting coordinates have the scale and orientation of the PC.

As is well known, WFPC2 detectors suffer from the charge transfer efficiency (CTE) problem which causes some signal to be lost when charge is transferred down the chip during readout. The required CTE corrections are performed by applying the prescriptions given by Dolphin (2000), in particular the Eqs. (5-9) and Tables 1 or 2 for cold $\left(-88^{\circ} \mathrm{C}\right)$ or warm $\left(-76{ }^{\circ} \mathrm{C}\right)$ camera observations respectively.

Since we are dealing with relatively crowded fields we decided to use the PSF-fitting photometry in the analytic approximation (a Penny function with four free parameters) as available with DAOPHOT II (Stetson 1987).

After matching the instrumental magnitudes to derive colours, we transformed our data to the Standard System following Dolphin (2000):

$$
\begin{aligned}
M_{\mathrm{std}}^{x} & =m_{\mathrm{PSF}}^{x}+2.5 \cdot\left[\log \left(t_{\mathrm{exp}}-25.0\right)\right]+Z P_{\mathrm{FS}} \\
& +\Delta Z_{\mathrm{CG}}+T 1 \cdot(C O L)_{\mathrm{std}}+T 2 \cdot(C O L)_{\mathrm{std}}^{2}
\end{aligned}
$$

where $m_{\mathrm{PSF}}^{x}$ refers to the instrumental magnitude of filter $x$ obtained with the DAOPHOT II package and estimated starting from an aperture of radius of $1 \mathrm{pxl} ; M_{\mathrm{std}}^{x}$ and $(C O L)_{\text {std }}$ are the standard system values; the coefficients $T 1, T 2$ and $Z P_{\mathrm{FS}}$ for $\mathrm{F} 450 \mathrm{~W}$ and $\mathrm{F} 555 \mathrm{~W}$ are taken from Table 7 and $\Delta Z_{\mathrm{CG}}$ from Table 5 of Dolphin (2000), whereas $t_{\text {exp }}$ is the exposure time.

The calibrated magnitudes are finally obtained by adopting the procedure outlined by Whitmore (1995) which can be summarized as follows:

$m^{x}=M_{\mathrm{std}}^{x}+C_{\mathrm{ap}}+C_{\mathrm{cont}}$.

The $\left(C_{\mathrm{ap}}\right)$ term is the aperture correction, calculated as the percentage of flux (counts) within an aperture radius $r=1$ pxl with respect to the "total" flux within $0.5^{\prime \prime}$. This correction is a mean value obtained from aperture photometry measurements over isolated stars. The values of the fluxes are listed in Tables $2 \mathrm{a}$ and $2 \mathrm{~b}$ of Holtzman et al. (1995a).

The effect of contaminants which adhere to the cold CCD windows of the WFPC2 are taken into account with the contamination term $C_{\text {cont }}$. According to Whitmore (1995), $C_{\text {cont }}$ shows a stable linear trend between two subsequent decontaminations:

$C_{\text {cont }}=-d \times t_{\text {dec }}$

where $t_{\text {dec }}$ represents the number of days after the last decontamination process, and $d$ the contamination rate as tabulated by Whitmore (1995). 


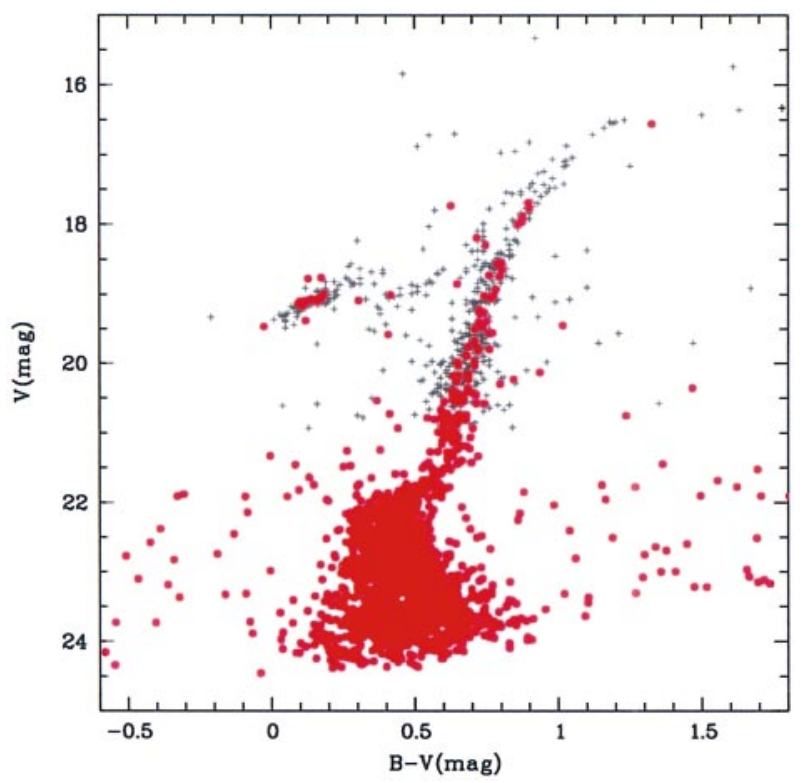

Fig. 2. NGC 2257: comparison between ground-based photometry selected from Walker (1989) data (cross) and our photometric results for the WF2 camera $(d o t)$.

In Fig. 1 we present an example of the typical photometric uncertainties as a function of magnitude for the cluster NGC 2155.

Following Sarajedini (1998), we evaluate the general validity of our reduction procedures by comparing our photometric results for the cluster NGC 2257 to the ground-based photometry from the work of Walker (1989). In particular, all of the CMD features appear to coincide within an observational uncertainty (Fig. 2). $( \pm 1 \sigma)$ :

The differences (our-Walker) in the zero points are $\Delta V=-0.01 \pm 0.04 \mathrm{mag}$

and

$\Delta(B-V)=0.02 \pm 0.03 \mathrm{mag}$.

A deeper analysis has been performed for the three LMC clusters in common with the work of Sarajedini (1998), i.e. NGC 2121, NGC 2155 and SL 663. The results of this comparison are summarized in Table 2. The agreement is relatively close, however, one may note that we find $V$ magnitudes systematically larger (from 0.03 mag up to $0.11 \mathrm{mag}$ ) and $B-V$ colours redder (up to $0.05 \mathrm{mag}$ ) than data by Sarajedini. The origin of these differences is not easily understood because of the different photometric techniques adopted. Moreover, we adopted the calibration procedure by Dolphin (2000) while Sarajedini follows Holtzman et al. (1995b), making it more puzzling to disentangle the origin of the quoted differences in the photometric data.

On this basis we assume that the data reduction method adopted for this work can be considered reliable at least within the range of the quoted value of uncertainty.

Crowding and incompleteness affect our analysis and are expected to be a function of magnitude and position in the frame. Tests using artificial stars are performed to infer

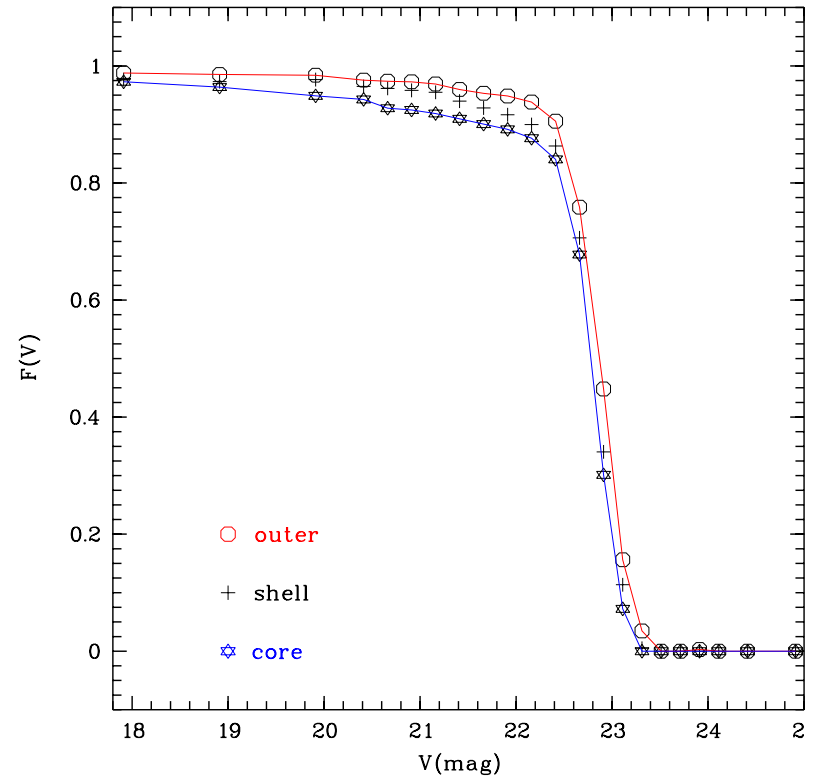

Fig. 3. Completeness curves for three circular regions (core: $R<R_{\mathrm{c}}$; shell: $1<R / R_{\mathrm{c}}<3$; outer: $R>3 R_{\mathrm{c}}$ ) around the centre of the cluster NGC 2155.

the completeness factors of the photometry. We generate the magnitudes of the input artificial stars by dividing our uncombined PC and WF LFs into bins of 0.1 magnitudes in $V$. In each bin 500 randomly distributed artificial stars have been added to the original frame. The photometric reduction is afterwards re-performed on the modified frame with exactly the same parameters previously used. Finally, we determine the fraction of the recovered stars. In order to quantify the crowding effect, we measure the completeness as a function of both position and $V$ magnitude for the same cluster. Figure 3 shows a set of completeness curves for three circular regions around the centre of the cluster NGC 2155. The magnitude for which the completeness factor is larger than 0.9 for all the selected rings is reported in the following paragraph (Table 3 ).

\section{The colour magnitude diagrams}

To provide a general view of the sample we are dealing with, the integrated magnitudes and colours of the clusters studied in this work are presented in Fig. 4 together with the data of 136 LMC clusters by Van den Bergh (1981). This figure shows that clusters in our sample represent quite well the main features of the distribution of the integrated colours and magnitudes of LMC clusters.

The spatial distribution in the LMC galaxy of the observed clusters is recalled in Fig. 5 .

The colour magnitude diagrams obtained for the 21 LMC (+1 SMC) star clusters and the surrounding fields are shown in Figs. 6-13 (left panels). These figures provide the photometric data of all the stars measured in the HST frames (PC + WFs) without applying any selection.

All the photometric data are available as ASCII files via WEB (http://www.te.astro.it/pub/lmc/). 
Table 2. Mean magnitudes and colours differences with Sarajedini (1998).

\begin{tabular}{ccccccc}
\hline Cluster & camera & $\langle\Delta V\rangle$ & $\sigma_{\Delta V}$ & $\langle\Delta(B-V)\rangle$ & $\sigma_{\Delta(B-V)}$ & common objects \\
\hline NGC 2121 & & -0.11 & $\mathbf{0 . 0 9}$ & $\mathbf{0 . 0 5}$ & $\mathbf{0 . 1 0}$ & \\
& PC & -0.10 & 0.07 & 0.05 & 0.09 & 820 \\
& WF2 & -0.10 & 0.10 & 0.05 & 0.11 & 1514 \\
& WF3 & -0.11 & 0.09 & 0.06 & 0.09 & 1117 \\
NGC 2155 & WF4 & -0.11 & 0.10 & 0.04 & 0.11 & 1433 \\
& & -0.06 & $\mathbf{0 . 0 8}$ & -0.01 & $\mathbf{0 . 1 0}$ & \\
& PC & -0.08 & 0.07 & 0.01 & 0.10 & 650 \\
& WF2 & -0.06 & 0.07 & -0.05 & 0.10 & 325 \\
& WF3 & -0.06 & 0.08 & 0.03 & 0.10 & 268 \\
& WF4 & -0.03 & 0.09 & -0.03 & 0.10 & 661 \\
& & -0.03 & $\mathbf{0 . 0 8}$ & $\mathbf{0 . 0 4}$ & $\mathbf{0 . 0 9}$ & \\
& PC & -0.04 & 0.06 & 0.04 & 0.10 & 335 \\
& WF2 & -0.01 & 0.08 & 0.04 & 0.09 & 400 \\
& WF3 & -0.03 & 0.08 & 0.02 & 0.08 & 338 \\
& WF4 & -0.03 & 0.08 & 0.05 & 0.10 & 527 \\
\hline
\end{tabular}
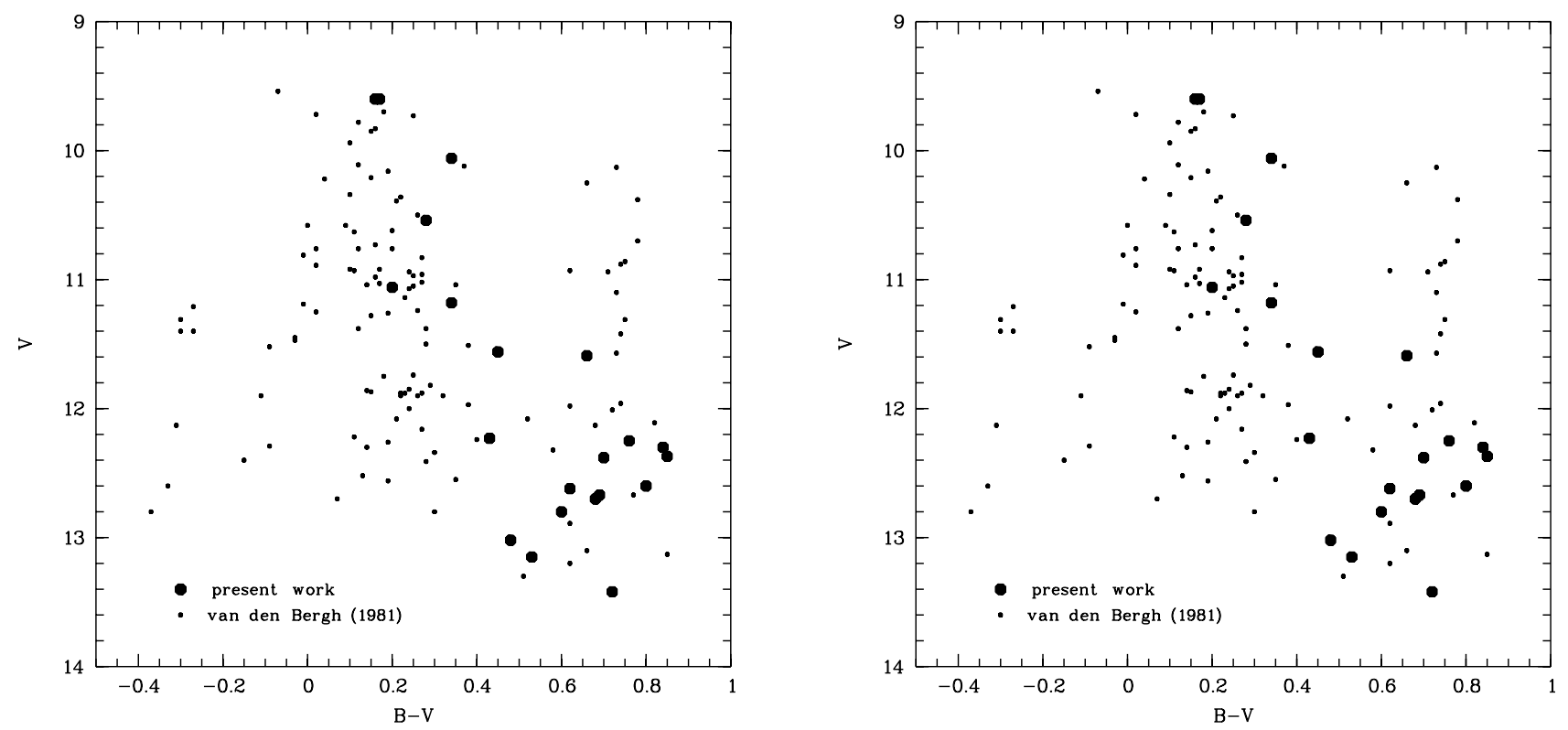

Fig. 4. The integrated properties of LMC clusters studied in this work are compared to a sample representative of the global cluster population of LMC.

The HST+WFPC2 resolution power is such that we are able to derive good photometric measurements also for the stars within $1 R_{\mathrm{c}}$ (defined as the radius for which the density in number of measured stars is half the central maximum). The CMDs of stars within $1 R_{c}$ are presented in Figs. 6-13 (right panels) and are also used to evaluate the CMD contamination from field stars.

Since a in-depth analysis of the derived CM for each cluster is beyond the aim of this paper, we briefly discuss only the main observational features and peculiarities.

\section{NGC 1651}

The CMD (2588 stars) discloses a MS termination $(V \sim$ $20.3)$ and the red giant clump (RGC) at $[V,(B-V)] \sim$ [19.25, 0.9]. A scarcely populated red giant branch (RGB) is present and also the subgiant branch (SGB) appears to be a feature of the cluster stars. The work by Mould et al. (1986) provides a much deeper CMD almost reaching $(V \sim 26)$ by using longer WFPC2 exposures. The core region is centred on the $\mathrm{PC}$.

\section{NGC 1718}

The CMD (3559 stars) shows a MS termination $(V \sim$ $20.5)$ and a well defined RGB together with a RGC at $[V,(B-V)] \sim[19.55,1.0]$. These features are more clearly visible in the inner regions where the contamination of field stars is less severe. The core region is centred on the PC. To our knowledge this is the first CMD of NGC 1718 appearing in literature. 


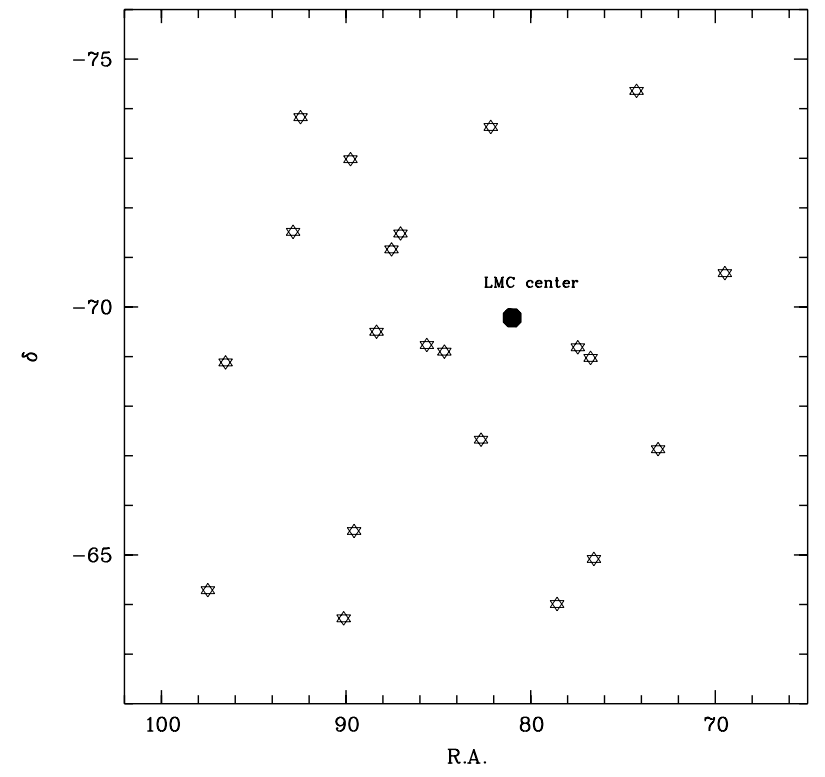

Fig. 5. Apparent spatial distribution of the studied LMC clusters (RA and $\delta$ units are degrees).

\section{NGC 1777}

The photometry of 2351 stars provides a CMD where the MS termination is at $V \sim 19.6$ and the RGC is at $[V,(B-V)] \sim[19.45,0.9]$. A populated RGB cannot be identified. The core region is centred on the $\mathrm{PC}$.

\section{NGC 1831}

The CMD of this cluster shows a well-populated MS ranging from $V \sim 18.3$ down to $V \sim 21.6$ and a red clump centred at $[V,(B-V)] \sim[18.45,0.9]$ in agreement with the results by Vallenari et al. (1992), but the present photometry is able to resolve the central region of the cluster providing measurements for 3934 stars. A slight trace of RGB could be recognized but the CMD of stars within $1 R_{\mathrm{c}}$ does not confirm this feature.

\section{NGC 1847}

Only $50 \%$ of the core of NGC 1847 is covered by the PC while the remaining core stars fall on WF2-4. However, a reliable CMD (2332 stars) discloses that the brighter MS stars are measured at $V \sim 15.9$ and some evidence of a small clump of red supergiants can be found at $[V,(B-$ $V)] \sim[14.8,1.4]$. The core He-burning red clump $[V,(B-$ $V)] \sim[19.5,1.0]$ and the imprint of a red giant branch are suggested to be CMD features due to the field star population. The field star contamination is particularly remarkable in this CM diagram since the cluster is located in the bar region of the LMC. Previous photometry was obtained by Nelson \& Hodge (1983).

\section{NGC 1856}

Again the cluster is not centred on the PC camera but the photometric data (6832 stars) largely improve on the previous photometry by Hodge \& Lee (1984). The MS reaches $V \sim 17.8$ and two red clumps can be identified at $[V,(B-V)] \sim[17.75,0.95]$ and $[V,(B-V)] \sim[19.5,1.0]$. The CMD of stars within $1 R_{\mathrm{c}}$ discloses that the first is due to the cluster stars and the second one to the field contamination. No evidence of a red giant branch is found in the CMD.

\section{NGC 1868}

The central region of this cluster is centred on the PC frame and the photometric data of 3344 stars disclose a well populated MS up to $V \sim 19.2$ and a red clump centred at $[V,(B-V)] \sim[19.0,0.75]$. A scarcely populated RGB (AGB?) appears to be present also in the CMD of the stars of the inner region. Previous photometry of this cluster by Corsi et al. (1994) did not reach the central region. Their determination of the $V_{\mathrm{TO}}$ is in good agreement with the present value.

\section{NGC 2004}

The CM diagram (1554 stars) of this cluster shows an extended main sequence (MS) ranging from $V \simeq 14$ down to $V=21.2$ and 5 red supergiants $(V \simeq 13.8,(B-V) \sim 1.4)$, the inspection of the CMD of stars within $1 R_{\mathrm{c}}$ (Fig. 8) shows that these features represent the cluster population. Also, the same figure discloses that the clump of red stars located at $V \simeq 19,(B-V) \sim 1$ is due to He-burning LMC field stars, as already suggested by Bencivenni et al. (1991). We note that four bright stars in the WFPC2 field, located at distances larger than $5 R_{\mathrm{c}}$, are saturated and we cannot provide their photometric measurements. However, the photometric data for these stars can be found in Balona \& Jerzykiewicz (1993) (their stars \# 54, 176, 201 and 321). Finally, a recent work by Keller et al. (2000) provides photometric results in three HST-WFPC2 filters (F160BW, F555W, F656N).

\section{NGC 2100}

Unfortunately, in the available images of this cluster the core is not located in the PC but just at the edge of the WF2. In this way at least $1 / 3$ of the cluster stars are missed because they are not in the field. Moreover, only a minor part of the core stars are measured taking advantage of the better PC resolution. The CM diagram (1726 stars) appears quite similar to that of NGC 2004, showing a MS termination at $V \sim 15.2$ and a group of red supergiants at $[V,(B-V)] \sim[13.8,1.7]$. Recent photometric data can be found in Keller et al. (2000) and Balona \& Jerzykiewicz (1993).

\section{NGC 2121}

From the CMD of all measured stars (5294) it is found that the MS termination $(V \sim 20.8)$ becomes quite similar 

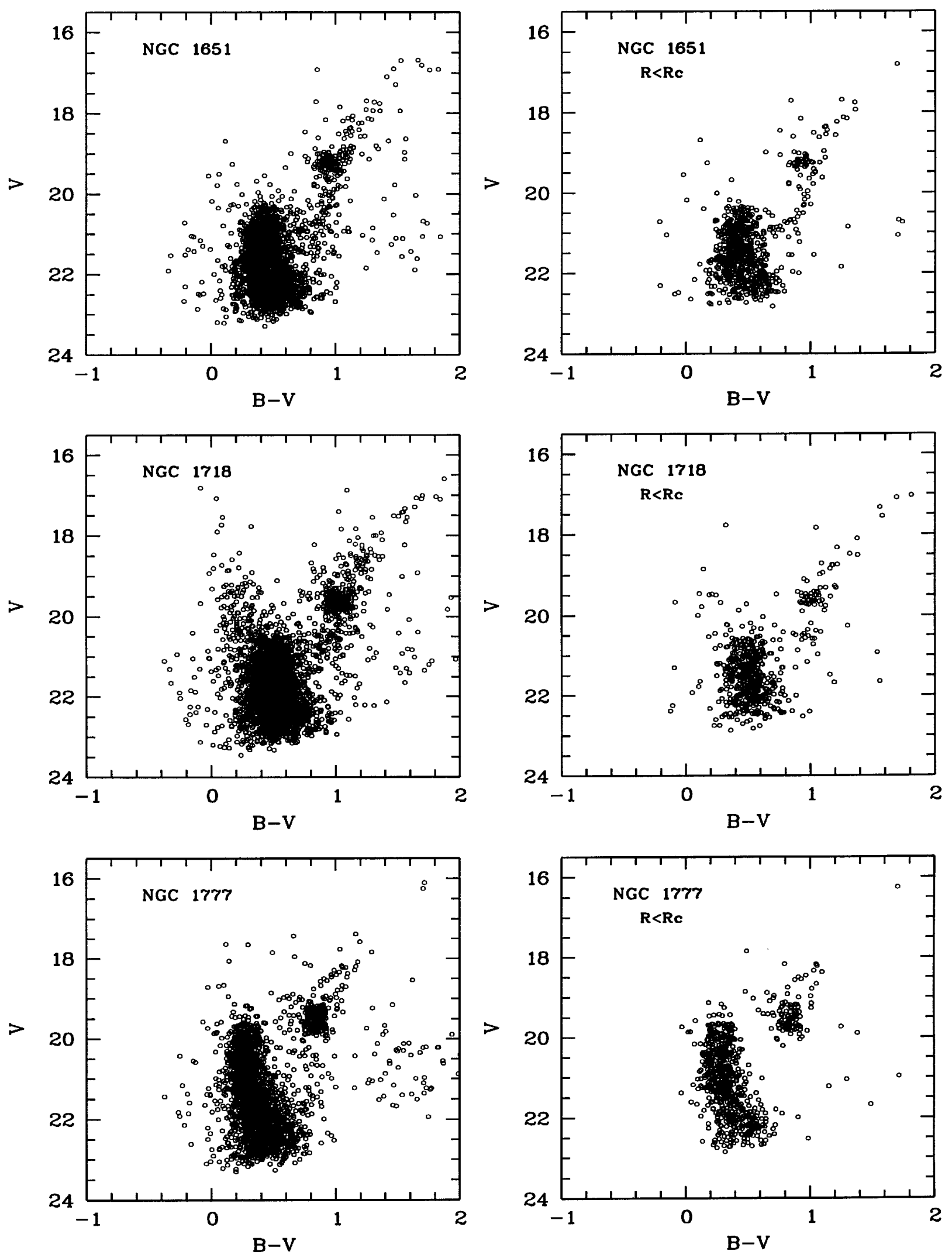

Fig. 6. Colour magnitude diagrams for three LMC clusters. The photometric data reported in the left panels refer to all the measured stars while the right panels show the stars located within $R \leq 1 R_{\mathrm{c}}$. 

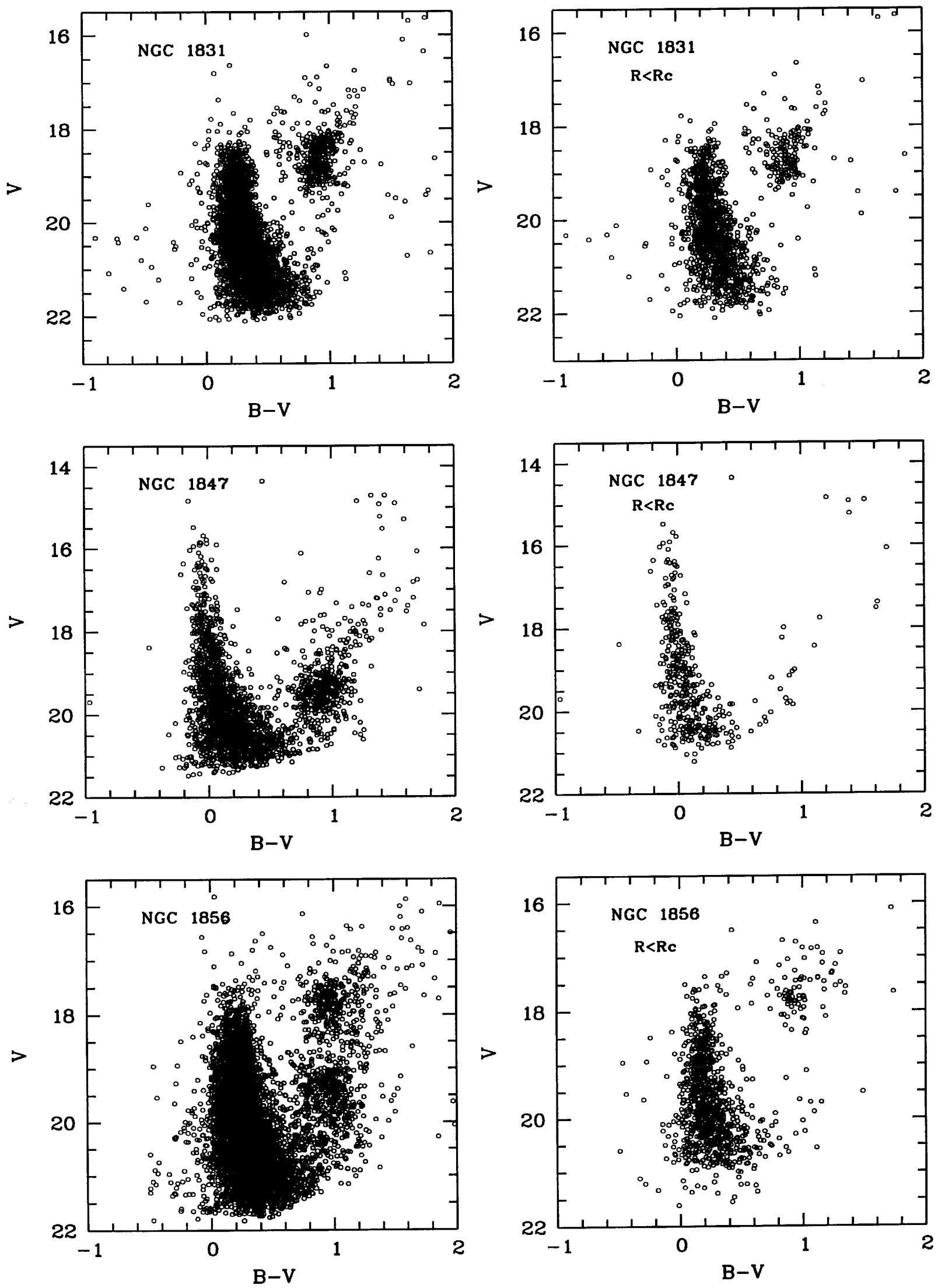

Fig. 7. As in Fig. 6 but for the three labelled clusters. 

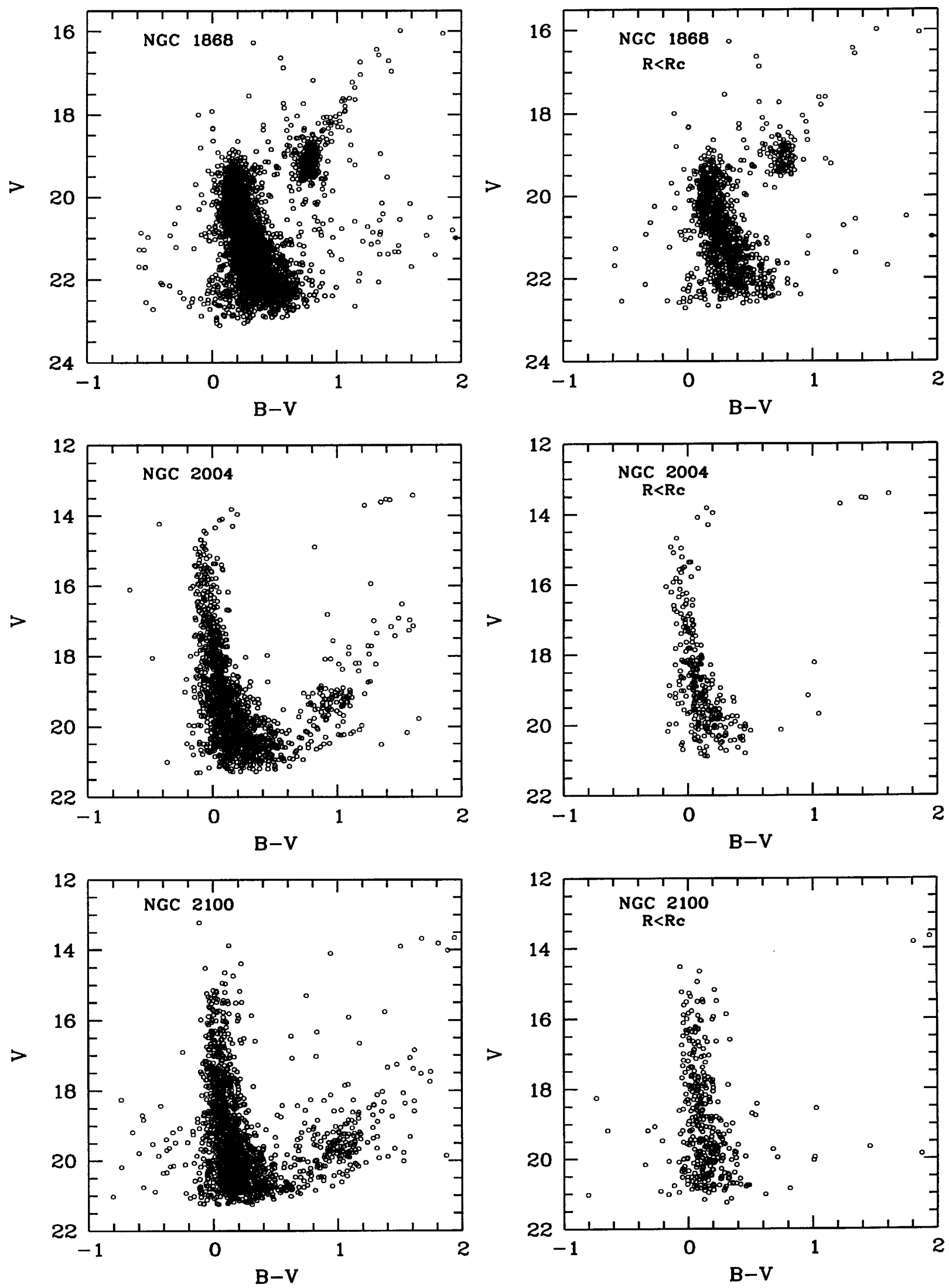

Fig. 8. As in Fig. 6 but for the three labelled clusters. 

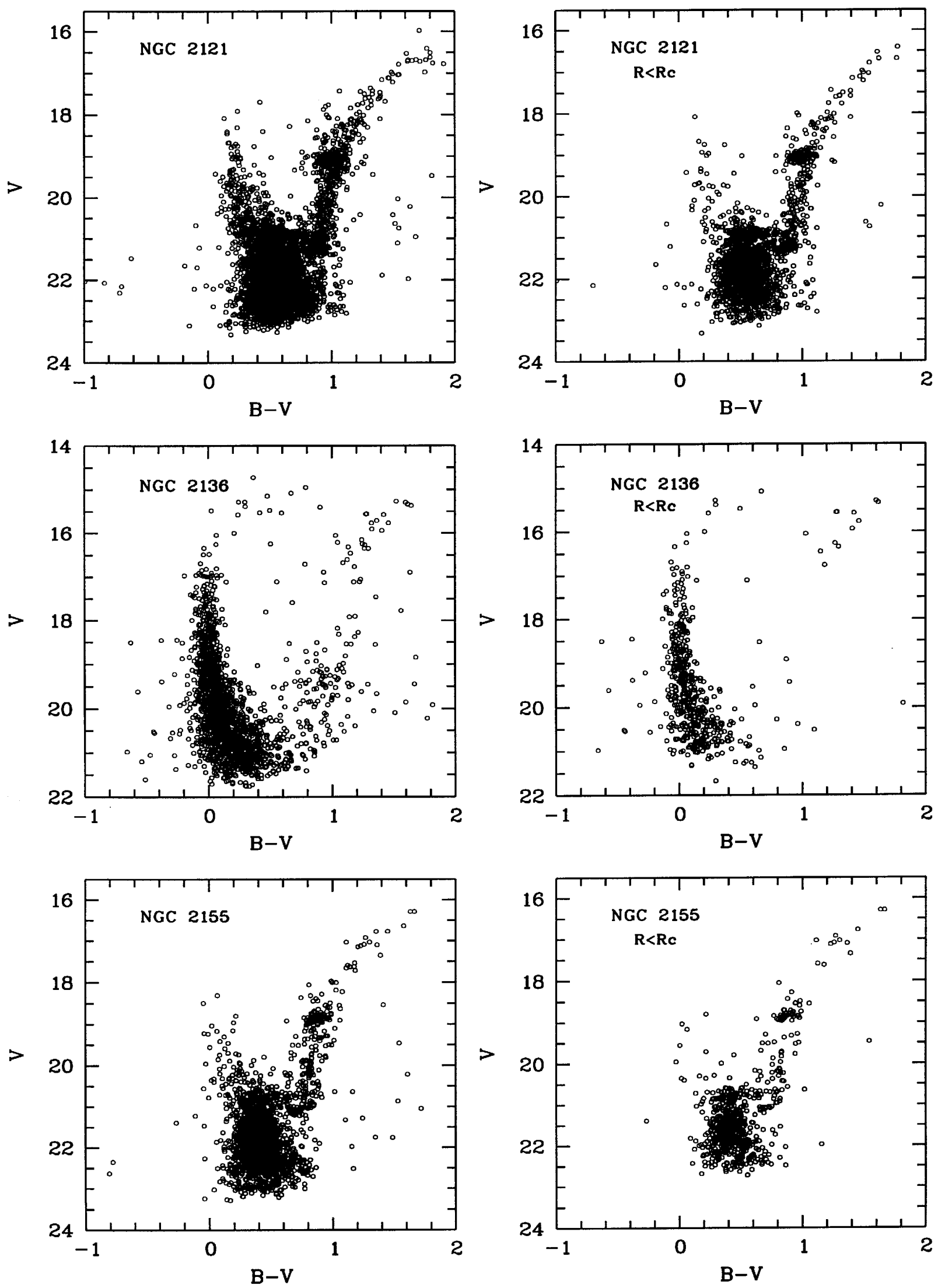

Fig. 9. As in Fig. 6 but for the three labelled clusters. 

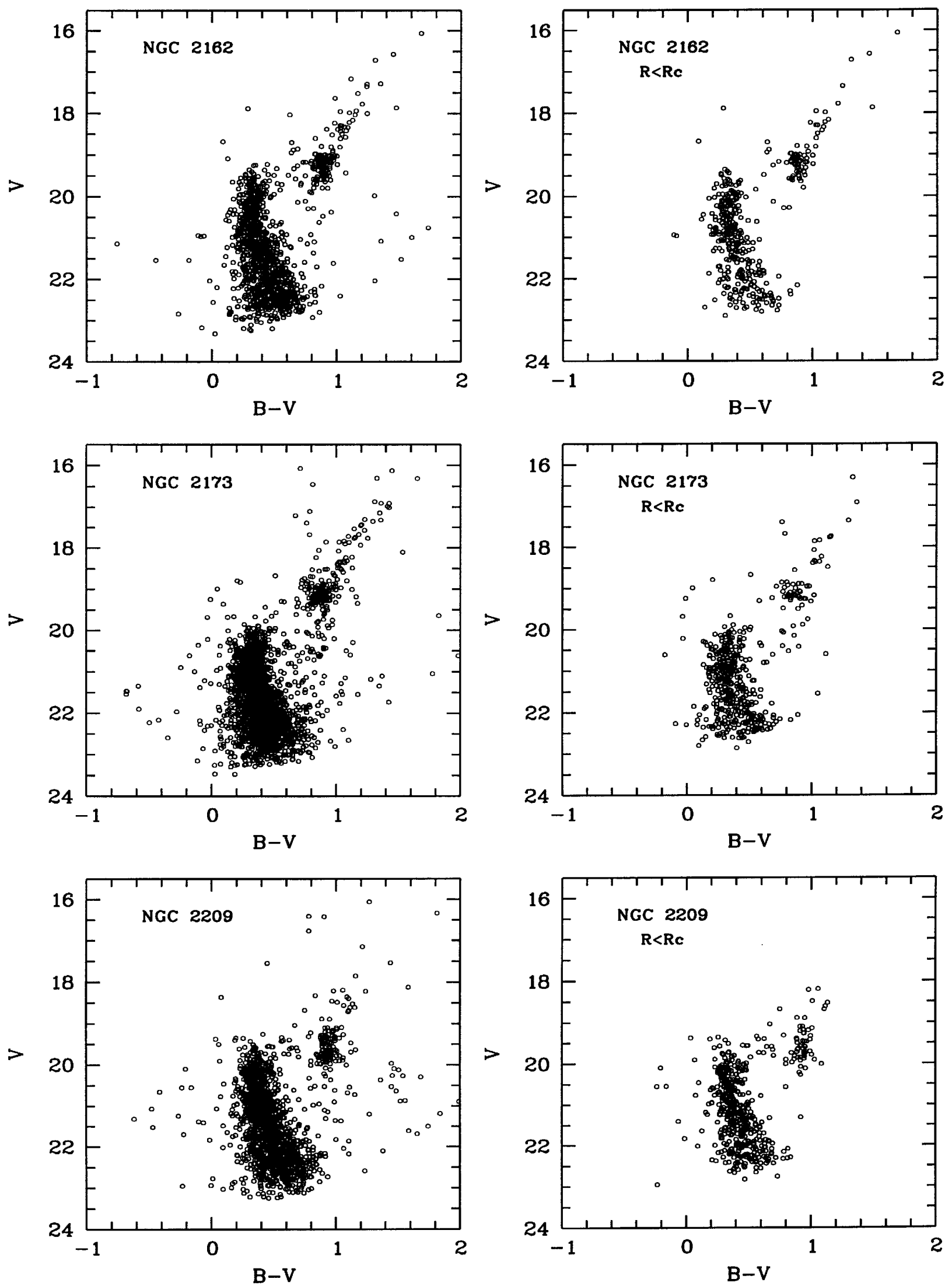

Fig. 10. As in Fig. 6 but for the three labelled clusters. 
E. Brocato et al.: Large Magellanic Cloud stellar clusters. I.
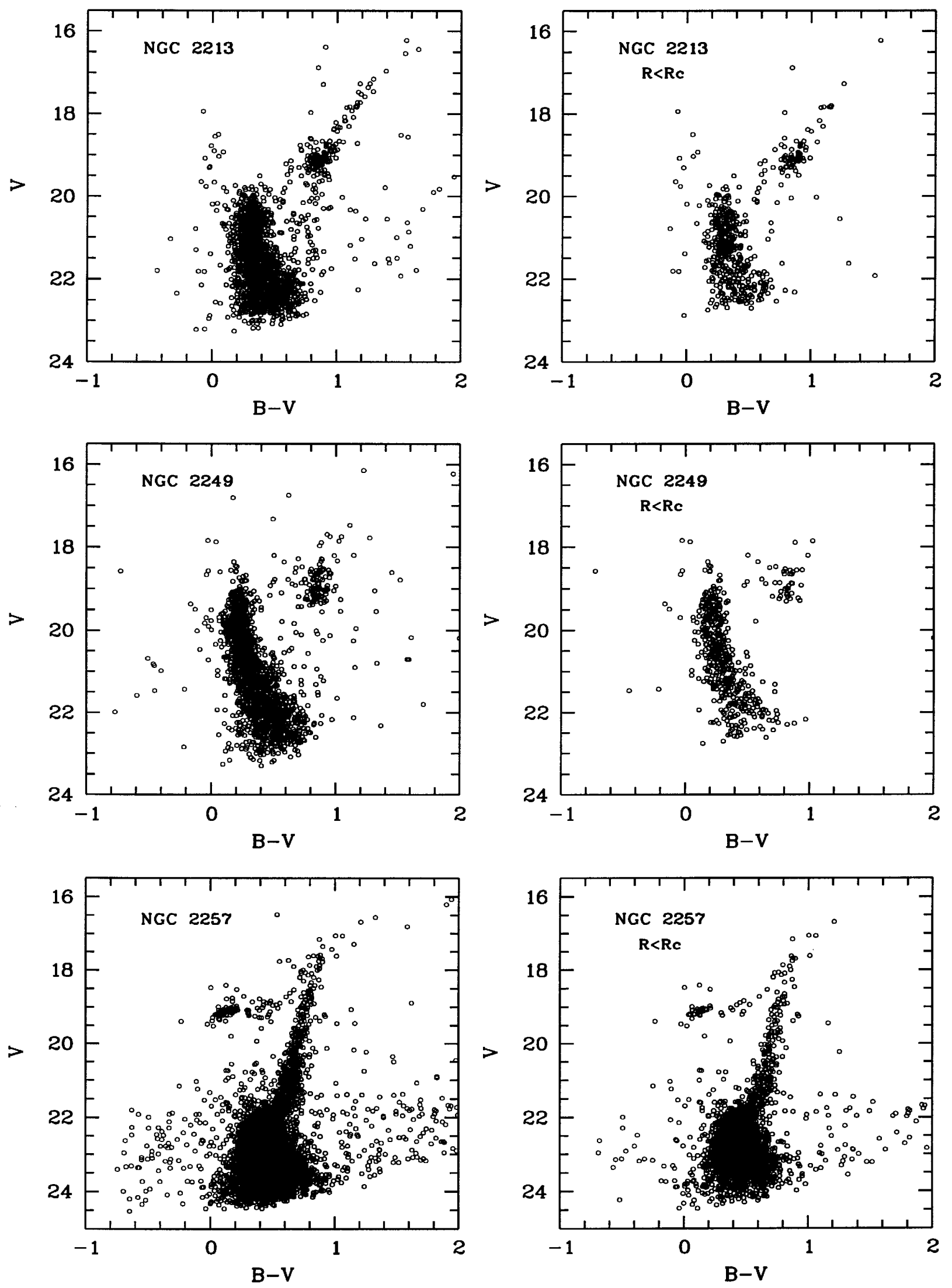

Fig. 11. As in Fig. 6 but for the three labelled clusters. 

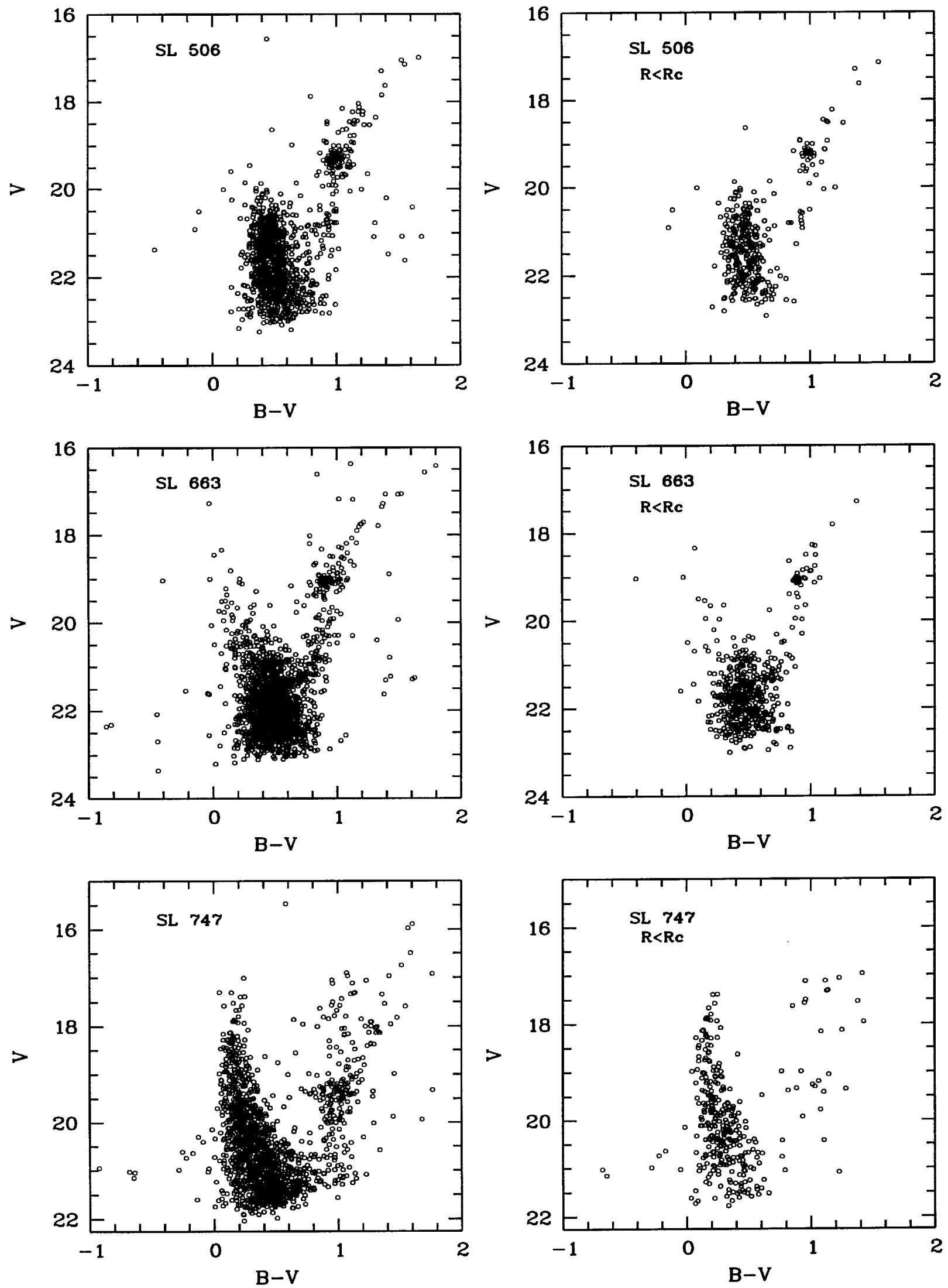

Fig. 12. As in Fig. 6 but for the three labelled clusters. 


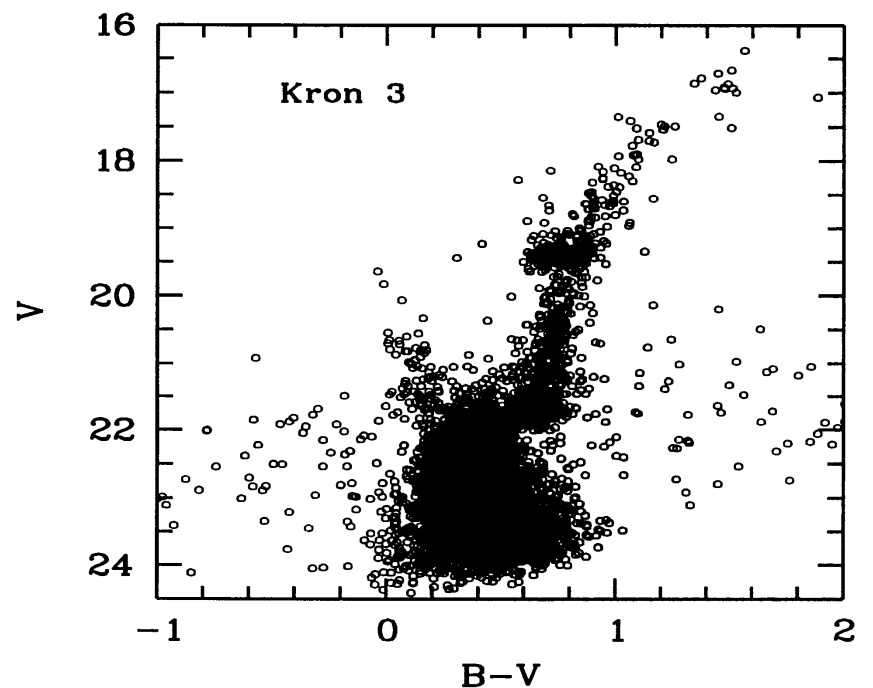

Fig. 13. As in Fig. 6 but for the Kron 3 cluster.

to the typical turn off (TO) of the galactic globular clusters. However, when plotted on a proper scale (Fig. 9) it is possible to recognize a group of stars lying just 0.5 mag above the TO. This feature cannot be clearly seen in the photometric results by Sarajedini (1998) (his Fig. 4) probably due to adopted size of the symbols. Previous groundbased photometry does not reach the magnitude required to see the TO (Flower et al. 1983). The RGC is located at $[V,(B-V)] \sim[19.05,1.0]$. The RGB and the SGB are well defined and populated. The core region is centred on the PC.

\section{NGC 2136}

The CMD (1758 stars) of this cluster shows an extended MS which, according to the luminosity function, appears to terminate at $V \sim 16.9$. A group of stars is located in the red (super)-giant region $V \leq 17$ and $(B-V) \geq 1$. Moreover, a number of bright blue stars populate the CMD at magnitude brighter than $V_{\text {TO }}$ with $(B-V)$ ranging from $\sim 0.0$ to $\sim 0.7$. The CMD of stars within $1 R_{\mathrm{c}}$ suggests that these stars belong to the cluster, so a deeper analysis is required to understand if we are dealing with i) stars experiencing the blue-loop during their core Heburning phase or ii) binaries or iii) stars captured from the nearby cluster companion NGC 2137 (see Hilker et al. 1995). The latter does not fall within the field of view of the present WFPC2 images. Previous $B V$ photometry can be found in Robertson (1974).

\section{NGC 2155}

The HST archive images of this cluster have been also studied by Sarajedini (1998) and the present CMD (2107 stars) does not improve significantly the previous one. The data are included in this work for the sake of homogeneity and also because a comparison with the Sarajedini results discloses a good agreement both for the

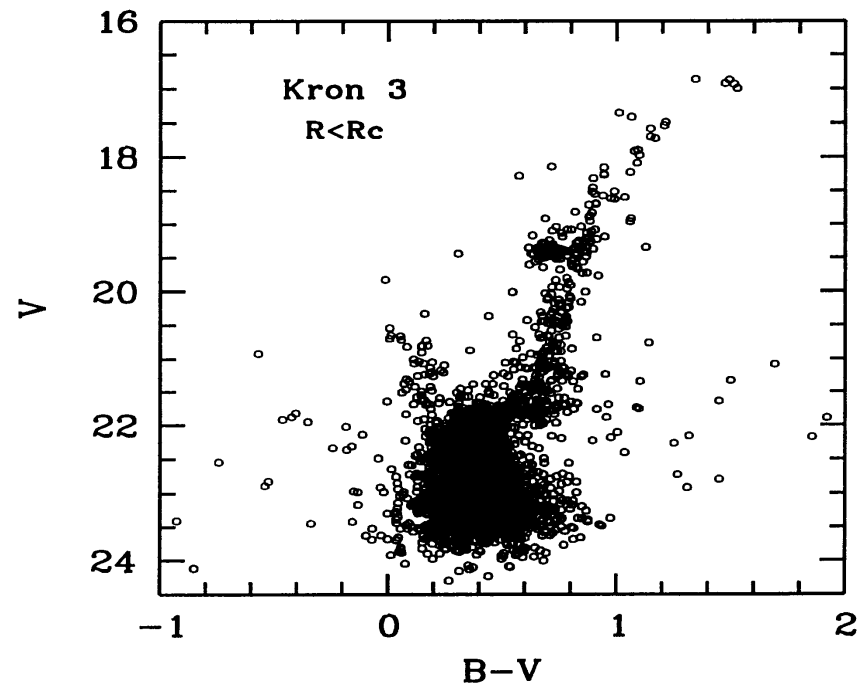

morphological features of the CMD and for the absolute calibration.

The TO is located at $V \sim 20.6$ and the RGB is clearly defined, and the RGC position is $[V,(B-V)] \sim$ $[18.85,0.9]$. The core region is centred on the PC.

\section{NGC 2162}

Ground based photometry for this cluster can be found in Corsi et al. (1994). The present CMD (1220 stars) shows a well defined MS with an upper termination at $V \sim 19.5$ and the RGC is at $[V,(B-V)] \sim[19.15,0.9]$. This CMD is particularly interesting since the Herzprung-Russell gap is well defined and the red portion of the CMD discloses the evidence of a well populated RGB. This cluster may recall the galactic open cluster NGC 2024 due to a small group of stars located just at a slightly brighter luminosity than $V_{\mathrm{TO}}$. The core region is centred on the PC.

\section{NGC 2173}

The CMD (2257 stars) discloses a MS termination $(V \sim$ $20.0)$ and the RGC at $[V,(B-V)] \sim[19.15,0.9]$. The RGB and the SGB are not well populated but visible. Previous works by Corsi et al. (1994) do not provide photometric data of the stars in the central region. The core region is centred on the PC.

\section{NGC 2209}

The CMD reports data for 1645 stars showing that the magnitude of the MS termination is at $V \sim 19.7$ and the red clump at $[V,(B-V)] \sim[19.35,0.95]$, in agreement with the photometry by Corsi et al. (1994). The CMD of stars in the central region discloses that the clump structure could be considered a "vertical structure" (VS) as identified by Piatti et al. (1999). 


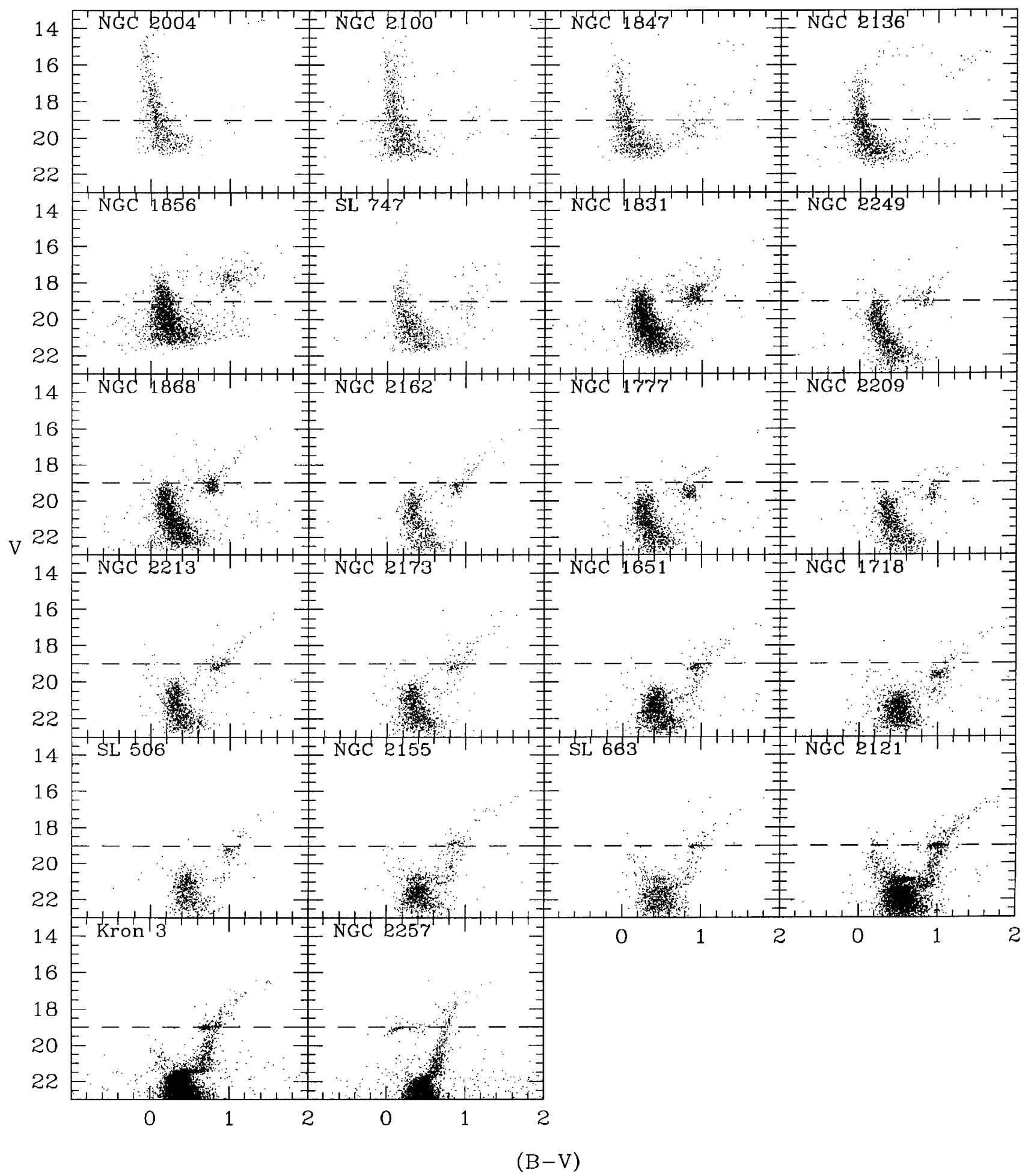

Fig. 14. Colour Magnitude diagrams for $21 \mathrm{LMC}$ clusters derived in the present work for stars with $R \leq 2 R_{\mathrm{c}}$. The dashed line at $V=19$ mag is displayed to make the "evolution" effect more visible along the cluster sequence. The SMC cluster Kron 3 is also shown assuming a magnitude shift of 0.4 mag to take into account the distance of SMC.

\section{NGC 2213}

The CMD (1900 stars) has a MS termination $(V \sim 19.9)$ and the RGC at $[V,(B-V)] \sim[19.15,0.9]$. The scarcely populated RGB is identified. A previous photometric investigation of this cluster has been performed by Da Costa et al. (1985). The core region is centred on the PC. 


\section{NGC 2249}

The cluster core is centred on the PC and the resulting CMD shows a well defined MS with an upper termination at $V \sim 19.0$. The red clump is located at $[V,(B-V)] \sim[18.7,0.9]$ and the RGB is not clearly established even considering the CMD in which all the measured star (1818) are included.

\section{NGC 2257}

This cluster has been widely studied in the past by several authors (see for example Walker 1989; Testa et al. 1995; Johnson et al. 1999); it is included in the present work as a template of the older globular clusters in the LMC. We note that the luminosity level of HB stars of our work is in good agreement with the HST results by Johnson et al. (1999), especially if one takes into account the differences in the exposure time of the observations (300 s against $3780 \mathrm{~s}$ in the $\mathrm{F} 555 \mathrm{~W}$ filter).

\section{SL 506}

The CMD (1102 stars) discloses a MS termination $(V \sim$ $20.5)$ and the RGC at $[V,(B-V)] \sim[19.25,1.0]$. A scarcely populated RGB is present but no SGB stars are found. Previous photometry (96 stars) on this cluster was performed by Jensen et al. (1988) in the $R$ and $G$ filters. The core region is centred on the $\mathrm{PC}$.

\section{SL 663}

The photometry of this cluster was recently performed by Sarajedini (1998) on the same HST data archive of the present work. The general agreement between the CMDs can be considered as a test on the consistency and reliability of the adopted data reduction procedures. Here we include measurements of 1834 stars. The CMD of this cluster discloses a turn off (TO) at $V \sim 20.7$, a subgiant branch (SGB) and a populated RGB. The red giant clump is at $[V,(B-V)] \sim[19.05,0.9]$. The core region is centred on the PC.

\section{SL 747}

The CMD (1554 stars) of this cluster discloses an extended MS which seems to terminate at $V \sim 18.2$. A group of stars is located in the red giant region $V \leq 17.2$ and $(B-$ $V) \geq 1.1$. To our knowledge this is the first CMD for this cluster. The core region is centred on the PC.

\section{Kron 3 (SMC)}

When we retrieved the data from the archive this cluster was originally marked as ESO 121-SC03, a deeper analysis on the pointing of the HST at the time of data acquisition disclosed that this dataset concerns the SMC cluster Kron 3. We decided to include anyway the CMD in this paper because it represent a simple stellar population of an age otherwise not present in this sample. This partially reduces the homogeneity of the sample when Kron 3 is included. However, we make clear that to compare Kron 3 with the other cluster presented here one needs to introduce corrections to take into account the distance and metallicity differences between LMC and SMC clusters. The CMD of the measured stars (8516) shows a well defined RGB and a RGC $(\mathrm{HB})$ at $[V,(B-V)] \sim[19.4,0.7]$. The TO point appears to be located at $[V,(B-V)] \sim$ $[22.2,0.4]$. The core region is centred on the PC. Recent photometric results have been presented by Rich et al. (2000) and Mighell et al. (1998) both the groups analyse the same HST dataset studied in the present work. In particular, Rich and collaborators provide a ridge-line which agrees with our data within $\delta V=0.1$ mag. For further and more exhaustive information about this cluster we address to the two quoted references.

Before closing this section let us show the data as arranged according to the observed upper MS termination.

This is done in Fig. 14, where one can clearly see the effect of stellar evolution from the top left panel down to the bottom right panel, i.e., along an age sequence from the younger to the older cluster. In Fig. 14 one may appreciate an explicit view of how a stellar population evolves with time. In good agreement with the theoretical expectations, the luminosity of the red giant clump decreases down to a minimum and then slightly increases again as soon as the electronic degeneracy of the He-core take place and the red giant branch appears. We note that some discontinuity in the RGC luminosity trend (i.e. NGC 2209 and NGC 2213) could be due to differences in the chemical composition of the clusters.

The same order of Fig. 14 is used in Table 3, where we also summarize the general features of the CM diagrams: in Col. 1 we report the name of the cluster, in Cols. 2 and 3 the Searle, Wilkinson \& Bagnuolo (1980) classification by Bica et al. (1996) and the age indicator ( $s$-parameter) by Elson \& Fall (1988) are reported, Cols. 4 and 5 represent magnitude and the colour of the MS termination (central $\mathrm{H}$ exhaustion), Cols. 6 and 7 are the location of the red clump (core He-burning stars), Col. 8 is the core radius $R_{\mathrm{c}}$ as previously defined, Col. 9 provides the magnitude limit of the measured stars, Col. 10 reports the completeness magnitude limit as defined in the previous paragraph. We note that the termination of the MS ( $\left.V_{\mathrm{TO}}\right)$ has been derived from the luminosity function following the indications given by Brocato \& Castellani (1987).

This table confirms that the SWB classes are well covered in our sample (only class II is missed), and this means that the clusters studied in this work span the wide range of age observable in the LMC cluster system.

Finally, we briefly remark the relation between the magnitude of the MS termination (which is a well known age indicator) and the observational age calibrations derived from the integrated colours of LMC clusters such as SWB and Elson \& Fall (1988). The agreement is quite satisfactory for the younger clusters, say up to SWB class IV. 
Table 3. Selected quantities for the LMC clusters of the studied sample. Evaluations with large uncertainties are marked by colon.

\begin{tabular}{cccccccccc}
\hline Cluster & SWB class & $s$-parameter & $V_{\mathrm{TO}}$ & $(B-V)_{\mathrm{TO}}$ & $V_{\mathrm{He}}$ & $(B-V)_{\mathrm{He}}$ & $R_{\mathrm{c}}(\operatorname{arcsec})$ & $V_{\text {lim }}$ & $V_{\text {lim }}^{\text {compl }}$ \\
\hline NGC 2004 & I & 15 & $14.6:$ & $-0.05:$ & $13.8:$ & $1.4:$ & 9.0 & 21.2 & 19.8 \\
NGC 2100 & I & 17 & $15.2:$ & $0.05:$ & $13.8:$ & $1.7:$ & 12.5 & 21.0 & 19.6 \\
NGC 1847 & I & 21 & $15.9:$ & $0.0:$ & $14.8:$ & $1.4:$ & 13.8 & 21.5 & 20.4 \\
NGC 2136 & III & 26 & 16.9 & 0.00 & $15.5:$ & $1.3:$ & 12.0 & 21.5 & 20.0 \\
NGC 1856 & IVA & 30 & 17.8 & 0.15 & 17.75 & 0.95 & 9.0 & 21.6 & 19.8 \\
SL 747 & IVA & - & 18.2 & 0.20 & $17.2:$ & $1.1:$ & 19.3 & 22.0 & 21.1 \\
NGC 1831 & IVA & 31 & $18.3:$ & $0.2:$ & 18.45 & 0.9 & 17.5 & 22.0 & 21.2 \\
NGC 2249 & IVB & 34 & 19.0 & 0.2 & 18.7 & 0.9 & 11.5 & 23.0 & 21.5 \\
NGC 1868 & IVA & 33 & 19.2 & 0.2 & 19.0 & 0.75 & 11.0 & 22.8 & 20.8 \\
NGC 2162 & V & 39 & 19.5 & 0.3 & 19.15 & 0.9 & 16.1 & 23.0 & 21.6 \\
NGC 1777 & IVB & 38 & 19.6 & 0.3 & 19.45 & 0.9 & 16.0 & 22.8 & 21.0 \\
NGC 2209 & IVB & 35 & 19.7 & 0.4 & 19.35 & 0.95 & 22.5 & 23.0 & 21.0 \\
NGC 2213 & V & 39 & 19.9 & 0.3 & 19.15 & 0.9 & 12.0 & 22.8 & 21.0 \\
NGC 2173 & VI & 42 & 20.0 & 0.3 & 19.15 & 0.9 & 12.5 & 23.2 & 21.2 \\
NGC 1651 & V & 39 & 20.3 & 0.4 & 19.25 & 0.9 & 17.5 & 22.8 & 21.2 \\
NGC 1718 & VI & 45 & 20.5 & 0.5 & 19.55 & 1.0 & 9.5 & 23.0 & 21.0 \\
SL 506 & V & 46 & 20.5 & 0.4 & 19.25 & 1.0 & 13.3 & 23.0 & $22.3:$ \\
NGC 2155 & VI & 45 & 20.6 & 0.4 & 18.85 & 0.9 & 16.1 & 23.0 & 21.8 \\
SL 663 & - & 36 & 20.7 & 0.5 & 19.05 & 0.9 & 20.7 & 23.0 & $22.2:$ \\
NGC 2121 & VI & 44 & $20.8:$ & 0.5 & 19.05 & 1.0 & 29.9 & 23.0 & $22.1:$ \\
NGC 2257 & VII & 51 & 22.4 & 0.4 & 19.05 & 0.1 & 35.5 & 24.0 & 20.5 \\
K3 SMC) & - & - & $22.2:$ & $0.4:$ & 19.4 & $0.7:$ & 20.7 & 24.0 & 21.8 \\
\hline
\end{tabular}

For older clusters we find some differences, in particular NGC 1651, NGC 2213 and NGC 2162 appear to have the same $s$-parameter (39) but the magnitudes for their MS terminations are ranging from 20.3 up to 19.5. Similarly, SL 663 is much older ( $\sim 3$ times) than what expected from the $s$-parameter calibration. Recalling that LMC stellar clusters are considered as templates for checking the integrated magnitude and colours expected by most of the population synthesis models, such a wide mismatch would require further future investigation to understand the nature of this apparent inconsistency (see however Ferraro et al. 1995).

\section{Conclusions}

In this paper we have presented photometric results for 21 LMC star clusters obtained from WFPC2 images retrieved from the HST archive. The very similar observational conditions obtained in space, the resolving power of the HST and the uniformity of the adopted data reduction procedures allow us to produce a homogeneous sample of CMD of a set of simple stellar populations of very different ages. The present sample certainly can be enlarged with more clusters to improve the completeness of the presented CMDs morphology but already it can be used to evaluate the evolutionary properties of simple stellar populations of ages ranging from a few Myr up to several Gyr. At the same time it is also a powerful database to investigate the evolution of the LMC stellar cluster system once a proper evaluation of the ages will be provided in a forthcoming paper. Finally, thanks to the large number of stars measured in each cluster, this sample is also recommended to probe the prediction of stellar evolutionary models in a wide range of masses (from $0.8 M_{\odot}$ up to $20 M_{\odot}$ ).

We intend to proceed with this project by improving the number of CMDs available for the LMC stellar cluster system but also extending these photometric studies to the SMC galaxy.

Acknowledgements. We warmly acknowledge Ata Sarajedini to address our attention on the mismatch in the archive between ESO 121-SC03 and Kron 3 and for kindly sending us the ASCII files of his photometric data of three clusters.

This work is supported by the Italian Ministry of University, Scientific Research and Technology (MURST): Cofin2000-Project: Stellar observable of cosmological relevance (MM02241491).

\section{References}

Arp, H. C. 1967, ApJ, 149, 91

Balona, L. A., \& Jerzykiewicz, M. 1993, MNRAS, 260, 782

Becker, S. A., \& Mathews, G. J. 1983 ApJ, 270, 155

Bencivenni, D., Brocato, E., Buonanno, R., \& Castellani, V. 1991, ApJS, 71, 109

Bica, E., Claria, J. J., Dottori, H., Santos, J. F. C. Jr, \& Piatti, A. E. 1996, ApJS, 102, 57

Brocato, E., \& Castellani, V. 1987, A\&A, 182, 36

Brocato, E., Buonanno, R., Castellani, V., \& Walker, A. R. 1989, ApJS, 71, 25

Brocato, E., Castellani, V., Ferraro, F. R., Piersimoni, A. M., \& Testa, V. 1996, MNRAS, 282, 614

Chiosi, C., Bertelli, G., Meylan, G., \& Ortolani, S. 1989, A\&A, 219,167 
Corsi, C. E., Buonanno, R., Fusi Pecci, F., et al. 1994, MNRAS, 271, 385

Da Costa, G. S., Mould, J. R., \& Crawford, D. 1985, ApJ, 297, 582

Dolphin, A. E. 2000, PASP, 112, 1397

Elson, R. A. W., \& Fall, S. M. 1988, AJ, 96, 1383

Ferraro, R. F., Fusi Pecci, F., Testa, V., et al. 1995, MNRAS, 272,391

Flower, P., Geisler, D., Schommer, R., Hodge, P., \& Olszewski, E. 1983, ApJ, 275, 15

Gascoigne, S. C. B. 1966, MNRAS, 134, 59

Hilker, M., Richtler, \& T., Stein, D. 1995, A\&A, 299, L37

Hodge, P. W. 1960a, ApJ, 132, 341

Hodge, P. W. 1960b, ApJ, 132, 346

Hodge, P. W., \& Lee, S.-O. 1984, ApJ, 276, 509

Holtzman, J. A., Hester, J. J., Casertano, S., et al. 1995a, PASP, 107, 156

Holtzman, J. A., Burrows, C. J., Casertano, S., et al. 1995b, PASP, 107, 1065

Iben, I. Jr. 1967, ARA\&A, 5, 57

Jensen, J., Mould, J., \& Reid, N. 1988, ApJS, 67, 77

Johnson, J. A., Bolte, M., Stetson, P. B., Hesser, J. E., \& Somerville, R. S. 1999, ApJ, 527, 199

Keller, S. C., Bessel, M. S., \& Da Costa, G. S. 2000, AJ, 119, 1748

Mateo, M. 1988, ApJ, 331, 261

Mighell, K. J., Sarajedini, A., \& French, R. S. 1998, AJ, 116, 2395
Mould, J. R., Da Costa, G. S., \& Wieland, F. P. 1986, AJ, 309, 39

Nelson, M., \& Hodge, P. 1983, PASP, 95, 5

Olsen, K. A. G, Hodge, P. W., Mateo, M., et al. 1998, MNRAS, 300,665

Piatti, A. E., Geisler, D., Bica, E., et al. 1999, AJ, 118, 2865

Rich, R. M., Shara, M., Fall, S. M., \& Zurek, D. 2000, AJ, 119, 197

Robertson, J. W. 1974, A\&AS, 15, 261

Sarajedini, A. 1998, AJ, 116, 738

Searle, L., Wilkinson, A., \& Bagnuolo, W. G. 1980, ApJ, 239, 803

Stetson, P. B. 1987, PASP, 99, 191

Testa, V., Ferraro, F. R., Brocato, E., \& Castellani, V. 1995, MNRAS, 275, 454

Van den Bergh, S. 1981, A\&AS, 46, 79

Vallenari, A., Aparicio, A., Fagotto, F., \& Chiosi, C. 1994, A\&A, 284, 424

Vallenari, A., Chiosi, C., Bertelli, G., Meylan, G., \& Ortolani, S. 1992, AJ, 104, 1100

Walker, A. R. 1989, AJ, 98, 2086

Walker, A. R. 1990, AJ, 100, 1532

Walker, A. R. 1992, AJ, 104, 1395

Walker, A. R. 1993a, AJ, 105, 527

Walker, A. R. 1993b, AJ, 106, 999

Whitmore, B. 1995, in Calibrating Hubble Space Telescope: Post Servicing Mission, ed. A. P. Koratkar, \& Leitherer C. (STScI, Baltimore), 269 
Online Material 
Table 1. Observations log.

\begin{tabular}{|c|c|c|c|c|}
\hline Cluster & Archive Directory/File & Filter & Exp. time (s) & Date (DD/MM/YY) \\
\hline \multirow{2}{*}{ NGC 1651} & u26m0axt/c0f_eaxw.fit & F450W & 230 & $01 / 02 / 94$ \\
\hline & u26m0ayh/c0f_eayk.fit & F555W & 120 & $01 / 02 / 94$ \\
\hline \multirow[t]{2}{*}{ NGC 1718} & u26m0aqb/c0f_eaqe.fit & F450W & 230 & $06 / 02 / 94$ \\
\hline & u26m0aqp/c0f_eaqs.fit & F555W & 120 & $06 / 02 / 94$ \\
\hline \multirow{2}{*}{ NGC 1777} & u26m0baz/c0f_ebbf.fit & F450W & 230 & $23 / 11 / 94$ \\
\hline & u26m0bbq/c0f_ebbw.fit & F555W & 120 & $23 / 11 / 94$ \\
\hline \multirow[t]{2}{*}{ NGC 1831} & u26m1bex/c0f_ebon.fit & F450W & 80 & $27 / 01 / 94$ \\
\hline & u26m1bez/c0f_eboz.fit & F555W & 40 & $27 / 01 / 94$ \\
\hline \multirow[t]{2}{*}{ NGC 1847} & u26m1bvy/c0f_ebwb.fit & F450W & 40 & $10 / 04 / 94$ \\
\hline & u26m1bwl/c0f_ebwp.fit & F555W & 20 & $10 / 04 / 94$ \\
\hline \multirow[t]{2}{*}{ NGC 1856} & u26m1bfj/c0f_ebrh.fit & F450W & 60 & $06 / 02 / 94$ \\
\hline & u26m1bru/c0f_ebrv.fit & F555W & 30 & $06 / 02 / 94$ \\
\hline \multirow[t]{2}{*}{ NGC 1868} & u26m0bet/c0f_ebnp.fit & F450W & 200 & $10 / 04 / 94$ \\
\hline & u26m0bev/c0f_ebob.fit & F555W & 100 & $10 / 04 / 94$ \\
\hline \multirow[t]{2}{*}{ NGC 2004} & u26m1bxz/c0f_ebyc.fit & F450W & 40 & $21 / 04 / 94$ \\
\hline & u26m1bym/c0f_ebyp.fit & F555W & 20 & $21 / 04 / 94$ \\
\hline \multirow[t]{2}{*}{ NGC 2100} & u26m1bwz/c0f_ebxc.fit & F450W & 40 & $21 / 04 / 94$ \\
\hline & u26m1bxm/c0f_ebxp.fit & F555W & 20 & $21 / 04 / 94$ \\
\hline \multirow{2}{*}{ NGC 2121} & u26m0bel/c0f_eblt.fit & F555W & 320 & $02 / 02 / 94$ \\
\hline & u26m0ben/c0f_ebmf.fit & F555W & 120 & $02 / 02 / 94$ \\
\hline \multirow[t]{2}{*}{ NGC 2136} & u26m1buf/c0f_ebui.fit & F450W & 60 & $11 / 02 / 94$ \\
\hline & u26m1bus/c0f_ebuv.fit & F555W & 30 & $11 / 02 / 94$ \\
\hline \multirow[t]{2}{*}{ NGC 2155} & u26m0ard/c0f_earg.fit & F450W & 230 & $01 / 02 / 94$ \\
\hline & u26m0bkj/c0f_ebkm.fit & F555W & 120 & $01 / 02 / 94$ \\
\hline \multirow[t]{2}{*}{ NGC 2162} & u26m0azx/c0f_ebaa.fit & F450W & 230 & $23 / 08 / 94$ \\
\hline & u26m0bal/c0f_ebao.fit & F555W & 120 & $23 / 08 / 94$ \\
\hline \multirow[t]{2}{*}{ NGC 2173} & u26m0asj/c0f_easn.fit & F450W & 230 & $02 / 02 / 94$ \\
\hline & u26m0asx/c0f_eatb.fit & F555W & 120 & $02 / 02 / 94$ \\
\hline \multirow[t]{2}{*}{ NGC 2209} & u26m0bdj/c0f_ebdm.fit & F450W & 230 & $01 / 02 / 94$ \\
\hline & u26m0bdx/c0f_ebea.fit & F555W & 120 & $01 / 02 / 94$ \\
\hline \multirow[t]{2}{*}{ NGC 2213} & u26m0bcl/c0f_ebcm.fit & F450W & 230 & $25 / 12 / 94$ \\
\hline & u26m0bcw/c0f_ebcz.fit & F555W & 120 & $25 / 12 / 94$ \\
\hline \multirow[t]{2}{*}{ NGC 2249} & u26m0bep/c0f_ebmr.fit & F450W & 230 & $11 / 08 / 94$ \\
\hline & u26m0ber/c0f_ebnd.fit & F555W & 120 & $11 / 08 / 94$ \\
\hline \multirow[t]{2}{*}{ NGC 2257} & u26m0alr/c0f_ealv.fit & F450W & 600 & $05 / 02 / 94$ \\
\hline & u26m0amf/c0f_eamj.fit & F555W & 300 & $05 / 02 / 94$ \\
\hline \multirow[t]{2}{*}{ SL 506} & u26m0atl/c0f_eatp.fit & F450W & 230 & $18 / 09 / 94$ \\
\hline & u26m0atz/c0f_eaud.fit & F555W & 120 & $18 / 09 / 94$ \\
\hline \multirow[t]{2}{*}{ SL 663} & u26m0art/c0f_earw.fit & F450W & 230 & $01 / 02 / 94$ \\
\hline & u26m0bkw/c0f_ebkz.fit & F555W & 120 & $01 / 02 / 94$ \\
\hline \multirow[t]{2}{*}{ SL 747} & u26m1bfb/c0f_ebpl.fit & F450W & 80 & $05 / 02 / 94$ \\
\hline & u26m1bfd/c0f_ebpx.fit & F555W & 40 & $05 / 02 / 94$ \\
\hline \multirow[t]{2}{*}{ K3 } & u26m0anv/c0f_eany.fit & F450W & 600 & $27 / 05 / 94$ \\
\hline & u26m0aol/c0f_eaoo.fit & F555W & 300 & $27 / 05 / 94$ \\
\hline
\end{tabular}

